\title{
Co-Existence of Motor Coordination Impairments in Semi- Hospitalized Children and Adolescents with Psychiatric Disorders
}

\author{
Margareth RM Dreyer ${ }^{1,3^{*}}$, Miguel A Boarati ${ }^{2,3}$, Telma Pantano ${ }^{2,3}$, Gabriel O Belizario ${ }^{4}$, Sandra \\ Scivoletto $^{2,3}$ and Cristiana CA Rocca ${ }^{1,3}$
}

${ }^{1}$ Serviço de Psicologia e Neuropsicologia, Instituto de Psiquiatria, Hospital das Clínicas HCFMUSP, Faculdade de Medicina, Universidade de São Paulo, São Paulo, SP, Brazil

${ }^{2}$ Instituto de Psiquiatria, Hospital das Clínicas HCFMUSP, Faculdade de Medicina, Universidade de São Paulo, São Paulo, SP, Brazil

${ }^{3}$ Hospital Dia Infantil, Instituto de Psiquiatria, Hospital das Clínicas HCFMUSP, Faculdade de Medicina, Universidade de São Paulo, São Paulo, SP, Brazil

${ }^{4}$ Programa de Transtorno Bipolar (PROMAN), Instituto de Psiquiatria, Hospital das Clínicas HCFMUSP, Faculdade de Medicina, Universidade de São Paulo, São Paulo, SP, Brazil

"Corresponding Author: Margareth R M Dreyer, Serviço de Psicologia e Neuropsicologia, Instituto de Psiquiatria, Hospital das Clínicas HCFMUSP, Faculdade de Medicina, Universidade de São Paulo, São Paulo, SP, Brazil, Tel: +5511 994410791; Email: mrmdreyer@ gmail.com

Received: 19 September 2017; Accepted: 26 September 2017; Published: 30 September 2017

\footnotetext{
Abstract

Background: Recent evidence suggests that children and adolescents presenting poor motor skills are at greater risk of developing internalizing symptoms, emotional/behavioural and externalizing problems. Motor coordination difficulties, as in Developmental Coordination Disorder, co-occurrence with Attention Deficit/Hyperactivity and Autism Spectrum Disorders are well documented. Few studies investigated the co-occurrence of motor difficulties and mood, anxiety, psychotic and emerging personality disorders in children and adolescents.

Objectives: To investigate whether children presenting psychiatric symptomatology manifest motor coordination difficulties.

Methods: 48 children and adolescents in semi-hospitalisation in a psychiatric-day facility were selected during a period of two years and screened according to DSM- 5 criteria and a motor coordination assessment battery. J Psychiatry Psychiatric Disord 2017; 1 (6): 294-303 
Results: Our findings demonstrated motor difficulties (percentile $\leq 16$ in Movement Assessment Battery for Children-2) to be significantly more prevalent among children and adolescents with Bipolar (15.62\%), Psychotic (9.37\%), Attention Deficit/Hyperactivity (21.87\%) and Autistic disorders (18.75\%). Motor coordination difficulties were equally prevalent among children presenting Anxiety (12.50\%), Conduct (3.12\%), Eating (3.12\%) and Depressive disorders $(9.37 \%)$ although no significant difference were detected between groups. Youth presenting traits of Emerging Personality Disorders did not reveal motor difficulties.

Conclusion: The co-occurrence of motor deficits and internalizing symptoms, such as in Bipolar and Psychotic disorders is somehow unexpected but offer evidence to support some previous findings in the literature. The presence of motor coordination deficits among patients with Attention deficit/ Hyperactivity and Autistic disorders is congruent to previous literature. Future research should further explore the association of motor deficits with Bipolar and Psychotic disorders in larger samples, and highlights the need for motor assessment in the practice of child psychiatry.

Keywords: Motor skills; Developmental coordination disorder; Child psychiatry disorder; Bipolar disorder; Neurodevelopmental disorders

\section{Introduction}

Developmental Coordination Disorder (DCD) affects 5-6\% of children between ages 5 and 11 years old with a male/female ratio between 2:1 and 7:1 according to the fifth Diagnostic and Statistical Manual of Mental Disorders DSM-5 [1]. There are reports in the literature suggesting associated impairments in children and adolescents with motor coordination difficulties. These impairments are related to self-esteem [2, 3], social adaptation [4], as well as cognitive [5] and learning processes [6] however, movement skill disorder is still an under-recognized condition (Wilson et al. 2012). The latest edition of DSM recognizes the pervasiveness in the DCD course, nevertheless there is a lack of identification of the condition either in adulthood or in childhood.

The current diagnostic of DCD is given when the acquisition and execution of coordinated motor skills is substantially below what is expected, given the individual's chronological age (criterion A); the disturbance significantly affects every day functioning (criterion B); the onset of symptoms is in the early developmental period (criterion C); the condition is not due to a general medical condition or intellectual disability (criterion D) [1].

There is a consensus that DCD is expected to co-occur with other neurodevelopmental disorders. The presence of DCD in children presenting Attention Deficit/Hyperactivity Disorder (ADHD) is well recognized (Sergeant 2000). Motor impairments are found to occur in $50 \%$ of the ADHD population. Motor coordination difficulties are also described in Autism Spectrum Disorder (ASD) [1] and Specific Learning Disorders [7].

Recently, a growing body of research focusing on motor skill difficulties in childhood suggests an association with psychiatric symptomatology, such as anxiety and depression. Some evidence has been reported suggesting that children and adolescents presenting with poor motor skills are at greater risk of developing internalizing symptoms [8]. Emotional/behavioural and externalizing problems have also been described in children and adolescents with 
motor skill problems [2]. Moreover, longitudinal studies have documented risks associated with psychiatric disorders in adults [9] and adolescents [10] who displayed poor motor skills during childhood.

In addition to the well know difficulties in carrying out activities of daily living, children with poor motor skills have been described in the literature as being affected by the negative impact of those impairments in other areas of children' and adolescents' lives such as academics, social and emotional domains. Some evidence has been described suggesting the co-existence of motor impairments and psychiatric symptoms in youth. In spite of the growing interest in exploring the association of internalizing and externalizing symptoms with motor impairments, there is a scarcity of quantitative reports in the literature about the existence and prevalence of those impairments in children and adolescents that have already developed symptoms of emotional deregulation, anxiety, mood and psychotic disorders. Hence, from a clinical perspective, it is important to investigate if this population of children and adolescents present motor coordination difficulties (and to which extent) so that those additional challenges they face can be taken in consideration in their treatment pathways.

To address the scarceness of research in this specific field, this study aimed to investigate the presence of motor coordination difficulties in a sample of children and adolescents presenting distinct symptoms of psychiatric disorders undergoing semi-hospitalization treatment.

\section{Materials and Methods}

A cross-sectional design was used in this study to describe the motor abilities of this population of children and adolescents. The sample was selected during a period of two years among all patients treated at the Child and Adolescent Day Hospital of Institute and Department of Psychiatry, of the University of São Paulo School of Medicine (IPq-HC-FMUSP). All patients undergoing a rehabilitation semi-hospitalization program were screened as part of the multidisciplinary diagnosis standard process of the day hospital. All parents or caregivers received an informed consent sheet from the Unit of Child and Adolescent Psychiatry (Serviço de Psiquiatria da Infância e Adolescência - SEPIA) from HC-FMUSP prior to testing and were free to decide whether or not to participate in the study. Only data from patients who affirmed they would agree to participate in the study were computed.

Inclusion criteria required participants to be 5 to 17 years and 06 months of age; have a diagnosis of severe mental disorder in a phase of exacerbation; present serious family and social conflicts that are responsible for clinical destabilization or worsening of evolution; be in a situation of risk or social vulnerability; present minimum impulse control capability; have availability to remain in semi-hospitalization; demonstrate minimum competence in activities of daily living and demonstrate failure in the outpatient approach. Individuals with Intellectual Disability (ID), presenting an IQ score below 70 in the Wechsler Intelligence Scales [11]; presenting any general medical conditions or mental disorders due to abuse of illicit drugs; neurological conditions (such as epilepsy, with neurological sequels due to head injury) and whose data were missing after the assessment were excluded from the study.

A trained psychologist employed the Movement Assessment Battery for Children- $2^{\text {nd }}$ Edition-MABC-2 [12] for the motor competence assessment, which evaluates aspects of gross and fine motor coordination, and static and dynamic 
balance. It is the most used battery to support DCD diagnosis worldwide and as such, facilitate motor abilities studies.

According to the European Academy for Childhood Disability [13], the study employed the $\leq 16^{\text {th }}$ percentile at the MABC2 as the cut-off score to classify the subjects within the "at risk for DCD" group, which included those whose motor performance is at or below the 5th percentile, representing children with DCD, provided that the other DSM-5 diagnostic criteria are met. Children presenting a score above the $16^{\text {th }}$ percentile were included in the "non-DCD" group.

All participants were evaluated for psychiatric diagnoses accordingly to the diagnostic criteria of the DSM-5, prevalence of diagnoses between groups were computed. For analysis purposes the sample was divided in groups of symptoms (Table 1):

\begin{tabular}{|c|c|}
\hline Diagnostic group & Diagnostics \\
\hline Anxiety Disorders (8) & $\begin{array}{l}\text { Social Anxiety disorder (1); Agoraphobia (4); Separation Anxiety } \\
\text { Disorder (1); Obsessive Compulsive Disorder (2) }\end{array}$ \\
\hline Feeding and Eating Disorders (1) & Anorexia nervosa (1) \\
\hline Psychotic Disorders (3) & $\begin{array}{l}\text { Unspecified Schizophrenia Spectrum and Other Psychotic Disorder } \\
\text { (1) Schizotypal (Personality) Disorder (1), Schizophrenia (1) }\end{array}$ \\
\hline Bipolar Disorders (5) & Bipolar disorder current episode hypomania (5) \\
\hline Depressive Disorders (8) & Depressive Disorders mild (7); Dysthymia (1) \\
\hline $\begin{array}{l}\text { Attention Deficit Hyperactivity Disorders } \\
\text { (10) }\end{array}$ & Attention-Deficit/Hyperactivity Disorder (10) \\
\hline $\begin{array}{l}\text { Disruptive, Impulse-Control and Conduct } \\
\text { Disorders (2) }\end{array}$ & Conduct disorder (2) \\
\hline Traits of Emerging Personality Disorders (3) & $\begin{array}{l}\text { Traits of Unspecified Personality Disorders (2); Traits of Borderline } \\
\text { Personality Disorder (1) }\end{array}$ \\
\hline Autism Spectrum Disorders (6) & Autism Spectrum Disorders (6) \\
\hline Other Mental Disorders (2) & $\begin{array}{l}\text { Traits of Somatic Symptom Disorder (1), Traits of Dissociative } \\
\text { Disorder (1) }\end{array}$ \\
\hline
\end{tabular}

Table 1: Distribution of diagnosis per groups.

Statistical analyses were performed using the IBM SPSS 21.0 statistical package.

A Student t-test, for continuum variables, and a Chi-square test, for categorical variables, were conducted to examine the presence of significant differences between groups on demographic variables. A Cochran-MantelHaenszel test, controlling for age, was employed to investigate the presence of significant different prevalence among the groups. 


\section{Results}

The final sample included 48 participants, consisting of 28 males and 20 females (58\% and $42 \%$ respectively). The age of the subjects ranged from 8 years and 3 months to 17 years and 11 months (mean age 12.59 and SD 2.91). Participants had an average of 7.25 years of schooling ( $S D=3.070)$, and a mean IQ score of $100.65(\mathrm{SD}=17.202)$.

Participants were evaluated using the MABC-2 and were divided into groups accordingly to their respective scores. 16 participants scored above the $16^{\text {th }}$ percentile and were allocated to the "Non-DCD" group (control group). 32 participants scored below or equal to the $16^{\text {th }}$ percentile and were allocated to the "At risk / presence of DCD" group (clinical group). In comparison with the "non- $D C D$ " group, the "at risk for $D C D$ " group consisted of younger children (mean age 11.71 SD 2.89) who had 3 school years less than the "non-DCD" group. Also, the "at risk for $D C D$ ” group presented Intelligence Quoefficient (IQ) scores 16 points lower in the Wechsler Intelligence Scales than the "non-DCD" group. After controlling for the IQ confounding factor, motor coordination differences found in this group of children and adolescents (with normal intelligence performance) were maintained, therefore suggesting that the IQ effect could not be accounted for the motor coordination deficits found. Usage of antipsychotic medication did not reveal significant differences between the groups (Table 2).

A Cochran-Mantel-Haenszel test, controlling for age and assessing significant differences between groups, revealed Bipolar disorders ( $\mathrm{p}<0.001)$, Psychotic disorders $(\mathrm{p}=0.012)$, ADHD $(\mathrm{p}<0.001)$, Autism spectrum disorder $(\mathrm{p}<0.001)$, and other mental disorders $(\mathrm{p}<0.001)$ to be significantly more prevalent in participants in the "at risk/presence of DCD” group. Emerging Personality Disorders $(\mathrm{p}=0.545)$, Anxiety disorders $(\mathrm{p}=0.290)$, Conduct disorders $(\mathrm{p}=0.459)$, Eating disorders $(\mathrm{p}=0.259)$ and Depressive disorders $(\mathrm{p}=0.633)$ were not significantly different between groups.

Except for the depressive diagnosis, "At risk / presence of DCD" subjects revealed an equal or higher prevalence in all psychiatric disorders assessed in this study.

\begin{tabular}{|l|l|l|}
\hline Variables & $\begin{array}{l}\text { Non-DCD } \\
(\mathbf{n = 1 6})\end{array}$ & $\begin{array}{l}\text { At risk/ Presence of DCD } \\
(\mathbf{n = 3 2})\end{array}$ \\
\hline Bipolar Disorders & $0(0)$ & $5(15.62)$ \\
\hline Depressive Disorders & $5(31.25)$ & $3(9.37)$ \\
\hline Traits of Emerging Personality Disorders & $3(18.75)$ & $0(0)$ \\
\hline Anxiety Disorders & $4(25.00)$ & $4(12.50)$ \\
\hline Psychotic Disorders & $0(0)$ & $3(9.37)$ \\
\hline ADHD & $3(18.75)$ & $7(21.87)$ \\
\hline ASD & $0(0)$ & $6(18.75)$ \\
\hline $\begin{array}{l}\text { Disruptive, Impulse Control and Conduct } \\
\text { Disorders }\end{array}$ & $1(6.25)$ & $1(3.12)$ \\
\hline Feeding and Eating Disorders & $0(0)$ & $1(3.12)$ \\
\hline Other Mental Disorders & $0(0)$ & $2(6.25)$ \\
\hline DCD, Devop &
\end{tabular}

DCD, Developmental Coordination Disorder; ADHD, Attention Deficit/Hyperactivity Disorder; Results expressed as frequency (\%) - Cochran-Mantel-Haenszel test; $\mathrm{p}<0.05$.

Table 2: Frequencies of psychiatric diagnoses between "at risk for DCD/presence of DCD" and "Non-DCD" subjects. 


\section{Discussion}

The majority of motor ability investigations associates motor impairments with neurodevelopmental disorders such as ADHD, ASD and learning disabilities and demonstrated the difficulties of the motor impaired children on carrying activities of daily living [1] or in social [4], and learning areas [7]. Recent evidence suggests associations with symptoms of internalizing and externalizing problems as well. Longitudinal studies have reported that there are more adults presenting internalizing symptoms such as depression and anxiety, who experienced motor difficulties in childhood, than those who weren't challenged during childhood.

To our knowledge, there are no reports of investigations of motor coordination abilities similar to this study, consisting of a clinical sample of children and adolescents with different diagnoses undergoing treatment for psychiatric disorders in a day-hospital facility during data collection.

We will examine the characteristics of the "Non-DCD" and the "At risk/presence of DCD" groups and afterwards we will give an overview of our motor abilities findings throughout the different psychiatric diagnoses. A more specific examination of the different motor findings throughout the distinct diagnoses falls outside the scope of this paper, which has an exploratory and observational nature of a clinical sample during treatment.

The motor ability assessments revealed that $67 \%(\mathrm{n}=32)$ of children and adolescents with psychiatric disorders in our sample were considered "at risk for DCD". The scores included participants' functioning in gross and fine motor coordination, and balance tasks. The lowest scores and most compromised aspect of their motor coordination was found to be at the gross motor skill component of the assessment (mean standard score 9.55 SD 4.99), followed by fine motor skill (mean standard score 11.95 SD 7.69) and balance component (mean standard score 22.75 SD 26.35). These results are congruent to Emck et al. [14] findings, which revealed mostly gross motor performance impairments in children with emotional, behavioral and pervasive developmental disorders.

As predicted, the presence of motor coordination difficulties in boys was higher than in girls. The male/female ratio (4:1) found in this study reflects the DCD prevalence, estimated to be between 2:1 and 7:1 [1]. Usage of antipsychotic medications among our sample, since it could interfere with motor performance, revealed no significant differences between the groups, suggesting that antipsychotic usage is not a confounding factor in our results. Likewise, motor coordination deficits could not be justified by IQ scores' differences, suggesting that children falling in the lower or superior IQ ranges were equally displaying motor coordination difficulties. This finding suggests that except for children with Intellectual Disability, who already have late motor developmental pathways, all children with average or above average intelligence could be at risk for DCD and thus, should be considered for proper screening and perhaps a more comprehensive motor skill assessment. Screening and assessment for motor skills should also be observed independently of age and years of schooling.

In accordance with previous literature [15], motor coordination difficulties were found in the participants presenting ADHD. The motor skill impairment rate of $70 \%$ observed in our sample of ADHD children was $20 \%$ higher than the average prevalence of DCD in ADHD cases, which is expected to be around 50\% and suggesting that the prevalence 
of motor impairments in children experiencing psychological distress, family and social conflicts is higher and could be associated to externalizing symptomatology. Also 100\% of children presenting ASD demonstrated motor impairments. Emck et al. [14] reported motor impairments and ASD to be highly intercorrelated, which was also found by Rinehart's et al. [16] and Nobile's et al. [17]. Their findings indicate that motor function impairment has a central role in ASD's presentation. Our results are congruent to previous literature, which suggests that both ADHD and ASD are often co-existent with motor skills impairments.

Regarding anxiety disorders, the present study found contrary results to previous literature, in which $50 \%$ of children, in our sample, presenting anxiety disorders also present motor skill impairments. However there was no significant difference between the groups. Moruzzi et al. [18] found a positive correlation between motor coordination difficulties, ADHD and anxiety symptoms in Italian twins between 8-17 years old. Several studies also highlighted the co-existence of motor skill deficits and anxiety disorders, including additional impairments, such as self perception of being less competent physically, being less accepted by peers [3] and having more social impairments [19].

This study also revealed contrary results to previous literature for the presence of motor skill impairments in children with depression. Previous findings from a cohort study [10] showed that boys with poor motor skills had 3 times more odds of maternally-rated anxiety at the age of 11 and 16 years than boys without motor coordination problems. Cairney's et al. [20] revealed an association between DCD and an increased risk of anxiety and depression in children. Our results regarding depressive disorders demonstrated only $37.5 \%$ of the children with depression were included in the "at risk for DCD group".

The most distinct observation from the data comparison was that motor coordination difficulties were found among $100 \%$ of bipolar disorder children and adolescents with current episode of hypomania.

There is evidence suggesting that BD's first episode occurrence in childhood and adolescence is associated with late neurodevelopmental processes, especially in cognitive functioning [21]. According to Lee et. al [21], in a metaanalysis, medium to large deficits in psychomotor speed were identified in first-episode BD. Negash et al. [22] and Dickenstein et al. [23] found that children with BD showed impaired sequential motor performance, which is consistent with the frequently reported impaired attention set shifting. Zhao et al. [24] also reported the existence of neurological abnormalities in sensory and motor performances in bipolar disorder. Finally, Mayoral et al. [25] found that motor impairments were more prevalent in early onset cases of both schizophrenia and bipolar disorder in comparison to healthy controls.

Our findings are congruent to previous evidence regarding motor impairments in children with psychotic conditions. The results revealed the presence of motor skill impairments among $100 \%$ of psychotic children and adolescents in our sample, and although the study employed a small sample size (3 children), which should be interpreted with caution, it is important to consider that the presence of neurological soft signs (clumsiness, motor incoordination, motor overflow, difficulty with motor sequencing or rapid successive movements) in the development of psychotic disorders has been well described in previous literature [26]. 
Finally, our results revealed an absence of motor coordination difficulties in children and adolescents presenting emerging personality disorder symptomatology. To our knowledge, no studies have reported any motor difficulties associated to this disorder, which the onset normally occurs later in adolescence and in young adults.

Our results are mostly congruent with previous literature and reinforce the hypothesis that children presenting psychiatric conditions should be evaluated for motor impairments. Except for children presenting traits of emerging personality disorders, all other psychiatric disorders included in the study were co-existent with motor skill impairments at least once, and Bipolar disorder, Psychotic disorder, ADHD and ASD were significantly more prevalent within this group.

\section{Limitations}

Firstly, the sample size was small and therefore the results should be interpreted with caution. Secondly, our sample consists of patients experiencing acute symptomatology that justify their inclusion in a semi-hospitalization program. It is possible that the motor difficulties found may be exacerbated during this period when therapeutic treatment is still being carried on and drug treatment is not always at its optimum level. Thus, further investigation should be conducted in a different phase of the presentation of symptoms.

Thirdly, despite the fact that those motor skills difficulties are revealed in tests, drawing attention to their coexistence with psychiatric symptomology, it is not possible to assume, from our results, that these children and adolescents, included in the "at risk for DCD group", need interventions to address those deficits, since we cannot affirm, as described in criterion B for the DCD diagnosis [1], that this impairment significantly interferes with their daily activities functioning or in their family, social, school, or community life. A proper diagnostic process should be conducted in order to determine that all criteria are met.

Finally, it is not within the scope of our study to examine motor coordination's relation with each diagnostic group, but to report an overview of motor coordination abilities throughout different psychiatric disorders in children and adolescents. Further data collection would be needed in order to address these study limitations.

\section{Conclusion}

The results revealed a higher prevalence of motor skills dysfunctions in the majority of children and adolescents undergoing treatment in a day hospital, suggesting that motor coordination problems co-exist in children and adolescents that are already experiencing psychological distress. Limited attention has been paid to motor impairments among children with early onset psychiatric disorders.

Despite the study's limitations, the results demonstrated novel and relevant evidence about prevalence of motor skill impairments in children with psychiatric disorders and should serve as a starting point to the development of further research aiming to investigate the course, impact and nature of the co-occurrence of motor coordination impairments and internalizing disorders symptomatology, especially bipolar and psychotic disorders in children and adolescents. 
Finally, considering the pervasiveness of the DCD course and the additional impairments' reports, our study highlights that, motor skills screening should become a routine procedure in mental health care facilities.

\section{Conflicts of Interest}

The authors have no conflicts of interest.

\section{References}

1. American Psychiatric Association. Diagnostic and statistical manual of mental disorders: DSM5 Washington: American Psychiatric Association (2013).

2. Emck C, Bosscher R, Beek P, et al. Gross motor performance and self-perceived motor competence in children with emotional, behavioural, and pervasive developmental disorders: a review. Developmental medicine and child neurology 51 (2009): 501-517.

3. Ekornås B, Lundervold AJ, Tjus T, et al. Anxiety disorders in 8-11-year-old children: motor skill performance and selfperception of competence. Scandinavian Journal of Psychology 51 (2010): 271-277.

4. Poulsen AA, Ziviani JM, Cuskelly M, et al. Boys with developmental coordination disorder: Loneliness and team sports participation. American Journal of Occupational Therapy 61 (2007): 451-462.

5. Piek JP, Dawson L, Smith LM, et al. The role of early fine and gross motor development on later motor and cognitive ability. Human movement science 27 (2008): 668-681.

6. Dewey D, Kaplan BJ, Crawford SG, et al. Developmental coordination disorder: Associated problems in attention, learning, and psychosocial adjustment. Human Movement Science 21 (2002): 905-918.

7. Flapper BC, Schoemaker MM. Developmental coordination disorder in children with specific language impairment: Co-morbidity and impact on quality of life. Research in developmental disabilities 34 (2013): 756-763.

8. Cairney J, Rigoli D, Piek J. Developmental coordination disorder and internalizing problems in children: the environmental stress hypothesis elaborated. Developmental Review 33 (2013): 224-238.

9. Hellgren L, Gillberg IC, Beagenholm A, et al. Children with deficits in attention, motor control and perception (DAMP) almost grown up: psychiatric and personality disorders at age 16 years. J Child Psychol Psychiatry 35 (1994): 1255-1271.

10. Sigurdsson E, Van Os J, Fombonne E. Are impaired childhood motor skills a risk factor for adolescent anxiety? Results from the 1958 U.K. birth cohort and the National Child Development Study. Am J Psychiatry 159 (2002): 1044.

11. Wechsler D. Manual for the Wechsler Preschool and Primary Scale of Intelligence. San Antonio, TX: The Psychological Corporation (1967).

12. Henderson SE, Sugden DA, Barnett AL. Movement assessment battery for children [examiner's manual] (2nd Edn.). London: Pearson Assessment (2007).

13. European Academy for Childhood Disability. EACD recommendations: 34 German-Swiss interdisciplinary clinical practice guideline. S3-standard according to the association of the scientific medical societies in Germany (AWMF). Revised for the UK. Turku, Finland: European Academy for Childhood Disability (2012). 
14. Emck C, Bosscher RJ, Van Wieringen PC, et al. Gross motor performance and physical fitness in children with psychiatric disorders. Developmental Medicine and Child Neurology 53 (2011): 150-155.

15. Gillberg C, Gillberg I, Rasmussen P, et al. Co- existinf disorders in ADHD-implications for diagnosis and intervention. Eur Child Adolesc Psychiatry 12 (2004): 80-92.

16. Rinehart NJ, Tonge BJ, Bradshaw JL, et al. Gait function in high-functioning autism and Asperger's disorder. European child \& adolescent psychiatry 15 (2006): 256-264.

17. Nobile M, Perego P, Piccinini L, et al. Further evidence of complex motor dysfunction in drug naive children with autism using automatic motion analysis of gait. Autism (2011): 1362361309356929.

18. Moruzzi S, Rijsdijk F, Battaglia M. A twin study of the relationships among inattention, hyperactivity/impulsivity and sluggish cognitive tempo problems. Journal of abnormal child psychology 42 (2014): 63-75.

19. Schoemaker MM, Kalverboer AF. Social and affective problems of children who are clumsy: How early do they begin? Adapted physical activity quarterly 11 (1994): 130-140.

20. Cairney J, Hay JA, Veldhuizen S, et al. Developmental coordination disorder, sex, and activity deficit over time: a longitudinal analysis of participation trajectories in children with and without coordination difficulties. Developmental Medicine \& Child Neurology 52 (2010): e67-e72.

21. Lee RS, Hermens DF, Scott J, et al. A meta-analysis of neuropsychological functioning in first-episode bipolar disorders. Journal of psychiatric research 57 (2014): 1-11.

22. Negash A, Kebede D, Alem A, et al. Neurological soft signs in bipolar I disorder patients. Journal of affective disorders 80 (2004): 221-230.

23. Dickstein DP, Garvey M, Pradella AG, et al. Neurologic examination abnormalities in children with bipolar disorder or attention-deficit/hyperactivity disorder. Biological Psychiatry 58 (2005): 517-524.

24. Zhao Q, Ma YT, Lui SS, et al. Neurological soft signs discriminate schizophrenia from major depression but not bipolar disorder. Prog Neuropsychopharmacol Biol Psychiatry 43 (2013): 72-78.

25. Mayoral M, Bombín I, Castro-Fornieles J, et al. Longitudinal study of neurological soft signs in firstepisode early-onset psychosis. J Child Psychol Psychiatry 53 (2012): 323-331.

26. Chan RC, Xie W, Geng FL, et al. Clinical Utility and Lifespan Profiling of Neurological Soft Signs in Schizophrenia Spectrum Disorders. Schizophrenia bulletin, sbv196 (2015).

(CC) This article is an open access article distributed under the terms and conditions of the 